UR-1344

ER-40685-793

\title{
TESTS OF INTEGRABILITY OF THE SUPERSYMMETRIC NONLINEAR SCHRÖDINGER EQUATION
}

\author{
J. C. Brunelli \\ and \\ Ashok Das \\ Department of Physics and Astronomy \\ University of Rochester \\ Rochester, NY 14627
}

\begin{abstract}
We apply various conventional tests of integrability to the supersymmetric nonlinear Schrödinger equation. We find that a matrix Lax pair exists and that the system has the Painlevé property only for a particular choice of the free parameters of the theory. We also show that the second Hamiltonian structure generalizes to superspace only for these values of the parameters. We are unable to construct a zero curvature formulation of the equations based on $\operatorname{OSp}(2 \mid 1)$. However, this attempt yields a nonsupersymmetric fermionic generalization of the nonlinear Schrödinger equation which appears to possess the Painlevé property.
\end{abstract}




\section{Introduction:}

The nonlinear Schrödinger equation is one of the most widely studied $1+1$ dimensional integrable systems [1-3]. In contrast, the supersymmetric nonlinear Schrödinger (SNLS) equation has not drawn as much attention. In fact, it was only very recently that the supersymmetrization of the nonlinear Schrödinger equation was proposed. It was shown in ref. 4 through a study of the prolongation structure [5] that there are two distinct supersymmetrizations - one with a free parameter - which are integrable. The structure of a supersymmetric theory is, in general, more restrictive than its bosonic counterpart $[6,7]$. We were, therefore, quite puzzled by the appearance of a free parameter in the supersymmetric nonlinear Schrödinger equation and decided to study this system more systematically. We find that various conventional tests of integrability select out only a supersymmetric system without any free parameter.

In sec. II, we briefly review the supersymmetric nonlinear Schrödinger equation both in components and in superspace. This also establishes our notations and conventions relative to ref. 4. In sec. III, we construct the matrix Lax pair for the supersymmetric equation in superspace and show that this is possible only if the supersymmetric equations have no free parameter. In sec. IV, we carry out the Painlevé analysis $[8,9]$ for the supersymmetric equation and we can construct a consistent set of solutions only for the case without any free parameters as in sec. III. In sec. V, we try to study the zero curvature formulation $[10,11]$ of the supersymmetric system. We show that it is not possible to derive these equations from a zero curvature condition based on $\operatorname{OSp}(2 \mid 1)[12,13]$. However, we can obtain a fermionic generalization very close in form to the supersymmetric equation without any free parameter. This fermionic generalization, even though not supersymmetric, seems to pass the Painlevé test and, therefore, we believe that this represents a new integrable system. In sec. VI, we analyze the Hamiltonian structures for the supersymmetric nonlinear Schrödinger equation and show that a second Hamiltonian structure exists only for the case without any free parameter. This is very similar to what happens 
in the analysis of the supersymmetric KdV equation. Finally, we present our conclusions in sec. VII.

\section{SNLS Equations:}

The nonlinear Schrödinger equation is described in terms of a complex bosonic variable $q(x, t)$ and has the form

$$
\begin{aligned}
i q_{t} & =-q_{x x}+2 k\left(q^{*} q\right) q \\
-i q_{t}^{*} & =-q_{x x}^{*}+2 k\left(q^{*} q\right) q^{*}
\end{aligned}
$$

Here the subscripts $t$ and $x$ refer to derivatives with respect to these variables. The parameter $k$ is real, arbitrary and its value can be set to unity by suitably rescaling the dynamical variables $q(x, t)$ and $q^{*}(x, t)$. A simple dimensional analysis shows that we can assign the following canonical dimensions to the various variables.

$$
\begin{array}{ll}
{[x]=-1} & {[t]=-2} \\
{[q]=\left[q^{*}\right]=1} & {[k]=0}
\end{array}
$$

The supersymmetric generalization of the nonlinear Schrödinger equation was proposed in ref. 4. In addition to the complex, bosonic variables $q(x, t)$, it also involves complex, fermionic variables $\phi(x, t)$ and has the form

$$
\begin{aligned}
i q_{t}=-q_{x x}+ & 2 k\left(q^{*} q\right) q+2 k(\alpha+\gamma) q^{*} \phi_{x} \phi-2 k \alpha q \phi \phi_{x}^{*} \\
& +2 k(2-2 \alpha-\gamma) q \phi_{x} \phi^{*}+2 k \gamma \phi \phi^{*} q_{x} \\
-i q_{t}^{*}=-q_{x x}^{*}+ & 2 k\left(q^{*} q\right) q^{*}+2 k(\alpha+\gamma) q \phi_{x}^{*} \phi^{*}-2 k \alpha q^{*} \phi^{*} \phi_{x} \\
& +2 k(2-2 \alpha-\gamma) q^{*} \phi_{x}^{*} \phi+2 k \gamma \phi^{*} \phi q_{x}^{*} \\
i \phi_{t}=-\phi_{x x} & +2 k \alpha q^{*} q \phi+2 k(1-\alpha) q^{2} \phi^{*}+2 k \gamma \phi \phi^{*} \phi_{x} \\
-i \phi_{t}^{*}=-\phi_{x x}^{*}+ & 2 k \alpha q^{*} q \phi^{*}+2 k(1-\alpha) q^{* 2} \phi+2 k \gamma \phi^{*} \phi \phi_{x}^{*}
\end{aligned}
$$

Comparing with the corresponding equations in ref. 4 it is easy to see that they are related by $q \rightarrow \sqrt{2} q, q^{*} \rightarrow \sqrt{2} q^{*}$ with the identifications

$$
\begin{aligned}
& c_{1}=2 k(\alpha+\gamma-1) \\
& c_{2}=2 k(\alpha-1)
\end{aligned}
$$


The set of equations (2.3) can be easily checked to be invariant under the supersymmetry transformations

$$
\begin{array}{ll}
\delta q=\epsilon \phi_{x} & \delta q^{*}=\epsilon \phi_{x}^{*} \\
\delta \phi=\epsilon q & \delta \phi^{*}=\epsilon q^{*}
\end{array}
$$

where $\epsilon$ is a real, constant Grassmann parameter.

A little bit of analysis shows that we can assign the following canonical dimensions to the various variables in Eq. (2.3).

$$
\begin{aligned}
& {[x]=-1 \quad[t]=-2} \\
& {[q]=\left[q^{*}\right]=1} \\
& {[\phi]=\left[\phi^{*}\right]=\frac{1}{2}} \\
& {[k]=[\alpha]=[\gamma]=0}
\end{aligned}
$$

The equations (2.3) can be put in a manifestly supersymmetric form by introducing a complex, fermionic superfield

$$
\Phi(x, t, \theta)=\phi(x, t)+\theta q(x, t)
$$

Here $\theta$ is the real, Grassmann coordinate of the superspace and by construction

$$
[\theta]=-\frac{1}{2} \quad[\Phi]=\frac{1}{2}
$$

Introducing the covariant derivative in superspace to be

$$
D=\frac{\partial}{\partial \theta}+\theta \frac{\partial}{\partial x}
$$

the equations in $(2.3)$ can be written also as

$$
\begin{gathered}
i \Phi_{t}=-D^{4} \Phi+2 k \alpha\left(D \Phi^{*}\right)(D \Phi) \Phi-2 k \gamma \Phi^{*} \Phi\left(D^{2} \Phi\right)+2 k(1-\alpha) \Phi^{*}(D \Phi)^{2} \\
-i \Phi_{t}^{*}=-D^{4} \Phi^{*}+2 k \alpha(D \Phi)\left(D \Phi^{*}\right) \Phi^{*}-2 k \gamma \Phi \Phi^{*}\left(D^{2} \Phi^{*}\right)+2 k(1-\alpha) \Phi\left(D \Phi^{*}\right)^{2}
\end{gathered}
$$

It is easy to see that this is the most general equation (with the appropriate phase invariance) in superspace (note $[D]=\frac{1}{2}$ ) which will reduce to the nonlinear Schrödinger 
equation in the bosonic limit. Even though the parameters $\alpha, \gamma$ (and, therefore, $c_{1}$ and $\left.c_{2}\right)$ are arbitrary, it was shown in ref. 4 that the system of equations (2.3) are integrable only if

$$
c_{1}=-4 k \quad c_{2}=0
$$

or

$$
c_{1}=c=\text { arbitrary } \quad c_{2}=4 k
$$

To further analyze integrability, we will construct the matrix Lax pair for the system of equations (2.3) in the next section.

\section{Matrix Lax Pair:}

The nonlinear Schrödinger equation, Eq. (2.1), can be written in terms of a Lax pair where the Lax operators are $2 \times 2$ matrices. Conventionally [14], it is well known that the Lax pair

$$
\begin{gathered}
L=\left(\begin{array}{cc}
i(1+\beta) \frac{\partial}{\partial x} & q^{*} \\
q & i(1-\beta) \frac{\partial}{\partial x}
\end{array}\right) \\
B=\left(\begin{array}{cc}
-i \beta \frac{\partial^{2}}{\partial x^{2}}+\frac{i q^{*} q}{1+\beta} & -q_{x}^{*} \\
q_{x} & -i k \frac{\partial^{2}}{\partial x^{2}}-\frac{i q^{*} q}{1-\beta}
\end{array}\right)
\end{gathered}
$$

where $k=\frac{2}{1-\beta^{2}}$, would give Eq. (2.1) from the Lax equation

$$
L_{t}=[L, B]
$$

It is quite straightforward to check that the Lax pair can be generalized to include a dimensionful parameter, $\zeta$. In fact, the pair

$$
\begin{gathered}
L=\left(\begin{array}{cc}
(1+\lambda) \frac{\partial}{\partial x}+i \zeta & \left(1-\lambda^{2}\right)^{1 / 2} k^{1 / 2} q^{*} \\
\left(1-\lambda^{2}\right)^{1 / 2} k^{1 / 2} q & (1-\lambda) \frac{\partial}{\partial x}+i \zeta
\end{array}\right) \\
B=\left(\begin{array}{cc}
-i \lambda \frac{\partial^{2}}{\partial x^{2}}+2 i \zeta^{2}-i k(1-\lambda) q^{*} q & -i\left(1-\lambda^{2}\right)^{1 / 2} k^{1 / 2} q_{x}^{*} \\
i\left(1-\lambda^{2}\right)^{1 / 2} k^{1 / 2} q_{x} & -i \lambda \frac{\partial^{2}}{\partial x^{2}}+2 i \zeta^{2}+i k(1+\lambda) q^{*} q
\end{array}\right)
\end{gathered}
$$


where $\lambda(\zeta)$ is an arbitrary dimensionless (dimensionful) parameter also give Eq. (2.1) from the Lax Eq. (3.2). Thus, there appears to be a certain degree of freedom in the choice of the matrix Lax pair for the nonlinear Schrödinger equation. However, we find that neither of the forms of the Lax pair in Eqs. (3.1) and (3.3) generalize to the supersymmetric case.

The most logical choice for the Lax pair, in the case of the supersymmetric theory, would be in terms of the superfields. To construct the Lax pair, we note that

$$
\begin{array}{ll}
{[L]} & =1 \quad[B]=2 \\
{[\Phi]=\left[\Phi^{*}\right]=\frac{1}{2}} &
\end{array}
$$

We write the most general ansatz, consistent with the canonical dimensions in Eq. (3.4), for $L$ and $B$ in terms of the superfields $\Phi, \Phi^{*}$ and the covariant derivative $D$. Requiring that the Lax equation

$$
L_{t}=[L, B]
$$

gives the manifestly supersymmetric equations in Eq. (2.10), we find that this is possible only if $\alpha=1=-\gamma$. In this case, the Lax pair has the form

$$
\begin{gathered}
L=\left(\begin{array}{cc}
\lambda D^{2}+i \zeta+\lambda k \Phi^{*} \Phi & \lambda k^{1 / 2}\left(D \Phi^{*}\right) \\
\lambda k^{1 / 2}(D \Phi) & \lambda D^{2}+i \zeta-\lambda k \Phi^{*} \Phi
\end{array}\right) \\
B=\left(\begin{array}{cc}
(i+\beta) D^{4}+\beta k\left(D \Phi^{*}\right)(D \Phi)+2 i \zeta^{2} & \beta k^{1 / 2}\left(D^{3} \Phi^{*}\right)+2(i+\beta) k^{1 / 2}\left(D \Phi^{*}\right) D^{2} \\
-i k\left(\left(D^{2} \Phi^{*}\right) \Phi-\Phi^{*}\left(D^{2} \Phi\right)\right) & \\
+(i+\beta) k\left\{\left(D^{2}\left(\Phi^{*} \Phi\right)\right)+2 \Phi^{*} \Phi D^{2}\right\} & \\
(2 i+\beta) k^{1 / 2}\left(D^{3} \Phi\right)+2(i+\beta) k^{1 / 2}(D \Phi) & (i+\beta) D^{4}+(2 i+\beta) k\left(D \Phi^{*}\right)(D \Phi) \\
& +2 i \zeta^{2}+i k\left(\left(D^{2} \Phi^{*}\right) \Phi-\Phi^{*}\left(D^{2} \Phi\right)\right) \\
& -(i+\beta) k\left\{\left(D^{2}\left(\Phi^{*} \Phi\right)\right)+2 \Phi^{*} \Phi D^{2}\right\}
\end{array}\right)
\end{gathered}
$$

where $\lambda$ and $\beta$ are arbitrary, dimensionless parameters and $\zeta$ is a dimensionful arbitrary parameter.

It is believed that an integrable system can be written as a Lax equation in terms of a pair of operators $L$ and $B$. The very fact that a Lax form of the equation exists only when 
$\alpha=1=-\gamma$ would then seem to indicate that the supersymmetric system is integrable only when there is no free parameter. (Incidentally, this choice of the parameters can be seen from Eq. (2.4) to imply that the system is integrable only for $c_{1}=-2 k, c_{2}=0$. This is very similar to Eq. (2.11) and we are hoping that there is a misprint in ref. 4). We also wish to point out that when $\alpha=1=-\gamma$, the supersymmetric equations have the form (omitting the complex conjugate equations)

$$
\begin{aligned}
i q_{t} & =-q_{x x}+2 k\left(q^{*} q\right) q-2 k q \phi \phi_{x}^{*}+2 k q \phi_{x} \phi^{*}-2 k \phi \phi^{*} q_{x} \\
i \phi_{t} & =-\phi_{x x}+2 k\left(q^{*} q\right) \phi-2 k \phi \phi^{*} \phi_{x}
\end{aligned}
$$

While the set of equations in Eq. (2.3) are invariant under the global phase transformations

$$
\begin{array}{ll}
q \rightarrow e^{-i \epsilon} q & q^{*} \rightarrow e^{i \epsilon} q^{*} \\
\phi \rightarrow e^{-i \epsilon} \phi & \phi^{*} \rightarrow e^{i \epsilon} \phi^{*}
\end{array}
$$

the set of equations in Eq. (3.6) have an invariance under a larger global transformation

$$
\begin{array}{ll}
q \rightarrow e^{-i \epsilon} q & q^{*} \rightarrow e^{i \epsilon} q^{*} \\
\phi \rightarrow e^{-i \sigma} \phi & \phi^{*} \rightarrow e^{i \sigma} \phi^{*}
\end{array}
$$

To understand integrability further, we turn to a Painlevé analysis of the system of equations in (2.3) in the next section.

\section{Painlevé Analysis:}

To carry out the Painlevé analysis [8,9] for the supersymmetric system in Eq. (2.3) we treat $q, q^{*}=p, \phi$ and $\phi^{*}=\psi$ as independent variables and rewrite the equations as

$$
\begin{aligned}
i q_{t}=-q_{x x}+ & 2 k(p q) q+2 k(\alpha+\gamma) p \phi_{x} \phi-2 k \alpha q \phi \psi_{x} \\
& +2 k(2-2 \alpha-\gamma) q \phi_{x} \psi+2 k \gamma \phi \psi q_{x} \\
-i p_{t}=-p_{x x}+ & 2 k(p q) p+2 k(\alpha+\gamma) q \psi_{x} \psi-2 k \alpha p \psi \phi_{x} \\
& +2 k(2-2 \alpha-\gamma) p \psi_{x} \phi+2 k \gamma \psi \phi p_{x} \\
i \phi_{t}=-\phi_{x x}+ & 2 k \alpha(p q) \phi+2 k(1-\alpha) q^{2} \psi+2 k \gamma \phi \psi \phi_{x} \\
-i \psi_{t}=-\psi_{x x}+ & 2 k \alpha(p q) \psi+2 k(1-\alpha) p^{2} \phi+2 k \gamma \psi \phi \psi_{x}
\end{aligned}
$$


Following the standard discussion [3], we choose a series expansion for the independent variables of the form

$$
\begin{aligned}
& q(x, t)=\xi^{\beta_{1}} \sum_{j=0}^{\infty} q_{j}(t) \xi^{j} \\
& p(x, t)=\xi^{\beta_{2}} \sum_{j=0}^{\infty} p_{j}(t) \xi^{j} \\
& \phi(x, t)=\xi^{\delta_{1}} \sum_{j=0}^{\infty} \phi_{j}(t) \xi^{j} \\
& \psi(x, t)=\xi^{\delta_{2}} \sum_{j=0}^{\infty} \psi_{j}(t) \xi^{j}
\end{aligned}
$$

where we assume the singularity surface of the solutions to have the form

$$
\xi(x, t)=x+f(t)=0
$$

We further assume that the zeroth order coefficients in the expansion in Eq. (4.2) do not vanish, namely,

$$
\begin{array}{ll}
q_{0} \neq 0 & p_{0} \neq 0 \\
\phi_{0} \neq 0 & \psi_{0} \neq 0
\end{array}
$$

Note also that while the coefficients $q_{j}(t)$ and $p_{j}(t)$ are bosonic, $\phi_{j}(t)$ and $\psi_{j}(t)$ are fermionic.

A leading order singularity analysis of Eq. (4.1) immediately gives

$$
\beta_{1}=\beta_{2}=\delta_{1}=\delta_{2}=-1
$$

Using these in Eq. (4.2) and substituting the series expansion into the dynamical equations in (4.1) yields the recursion relations between the coefficients. These can be written compactly in the matrix notation as

$$
M \chi=F
$$

where

$$
\chi=\left(\begin{array}{c}
q_{j}(t) \\
p_{j}(t) \\
\phi_{j}(t) \\
\psi_{j}(t)
\end{array}\right)
$$




$$
F=\left(\begin{array}{c}
F_{j}(t) \\
\tilde{F}_{j}(t) \\
G_{j}(t) \\
\tilde{G}_{j}(t)
\end{array}\right)
$$

and the supermatrix $M$ has the form

$$
M=\left(\begin{array}{ll}
A & D \\
C & B
\end{array}\right)
$$

and $A, B, C, D$ are $2 \times 2$ matrices. The matrices $A$ and $B$ have the form

$$
A=\left(\begin{array}{cc}
-(j-1)(j-2)+4 k q_{0} p_{0} & 2 k q_{0}^{2}+2 k(\alpha+\gamma) \phi_{1} \phi_{0} \\
+2 k(\alpha+(j-1) \gamma) \phi_{1} \psi_{0} & \\
+2 k(2(\alpha-1)+j \gamma) \phi_{0} \psi_{1} & \\
2 k p_{0}^{2}+2 k(\alpha+\gamma) \psi_{1} \psi_{0} & -(j-1)(j-2)+4 k q_{0} p_{0} \\
& +2 k(\alpha+(j-1) \gamma) \psi_{1} \phi_{0} \\
& +2 k(2(\alpha-1)+j \gamma) \psi_{0} \phi_{1}
\end{array}\right)
$$

and

$$
B=\left(\begin{array}{cc}
-(j-1)(j-2)+2 k \alpha q_{0} p_{0} & 2 k(1-\alpha) q_{0}^{2}+2 k \gamma \phi_{1} \phi_{0} \\
+2 k \gamma(1-j) \psi_{0} \phi_{1}-2 k \gamma j \psi_{1} \phi_{0} & \\
2 k(1-\alpha) p_{0}^{2}+2 k \gamma \psi_{1} \psi_{0} & -(j-1)(j-2)+2 k \alpha q_{0} p_{0} \\
& +2 k \gamma(1-j) \phi_{0} \psi_{1}-2 k \gamma j \phi_{1} \psi_{0}
\end{array}\right)
$$

We note that Eq. (4.1) describes a set of four coupled second order equations and, therefore, we expect eight arbitrary coefficients for a unique solution of the system. The arbitrariness will arise whenever the matrix relations in Eq. (4.6) are not invertible. For a supermatrix $M$ of the form in Eq. (4.9), this happens when [15]

$$
\operatorname{det} A=0
$$

and

$$
\operatorname{det} B=0
$$

From the forms of the matrices $A$ and $B$ in Eqs. (4.10) and (4.11) we note that 


$$
\begin{aligned}
\operatorname{det} A= & \left((j-1)(j-2)-2 k q_{0} p_{0}\right)\left((j-1)(j-2)-6 k q_{0} p_{0}\right) \\
& +2 k(\alpha+\gamma-2)\left((j-1)(j-2)-4 k q_{0} p_{0}\right)\left(\psi_{1} \phi_{0}-\psi_{0} \phi_{1}\right) \\
& -4 k^{2}(\alpha+\gamma)\left(q_{0}^{2} \psi_{1} \psi_{0}+p_{0}^{2} \phi_{1} \phi_{0}\right) \\
\operatorname{det} B= & \left((j-1)(j-2)+2 k(1-2 \alpha) q_{0} p_{0}\right)\left((j-1)(j-2)-2 k q_{0} p_{0}\right) \\
+ & 2 k \gamma\left((j-1)(j-2)-2 k \alpha q_{0} p_{0}\right) \psi_{1} \phi_{0} \\
+ & 2 k \gamma\left((j-1)(j-2)-2 k \alpha q_{0} p_{0}\right) \phi_{1} \phi_{0} \\
& -4 k^{2} \gamma(1-\alpha)\left(q_{0}^{2} \psi_{1} \psi_{0}+p_{0}^{2} \phi_{1} \phi_{0}\right)
\end{aligned}
$$

A low order analysis of the recursion relations gives

$$
q_{0} p_{0}=\frac{1}{k}
$$

as in the bosonic nonlinear Schrödinger equation as well as

$$
\psi_{0} \propto \phi_{0}
$$

Requiring $\operatorname{det} A$ and $\operatorname{det} B$ to vanish, we can determine the resonances. (These are the $j$-values at which arbitrary coefficients can arise.) From the bosonic part of $\operatorname{det} A$ we obtain

$$
j=-1,0,3,4
$$

while the vanishing of the bosonic part of $\operatorname{det} B$ gives
i) $\alpha=\frac{1}{2} \quad j=0,1,2,3$
ii) $\alpha=1 \quad j=0,0,3,3$

The expressions in the determinants containing the fermionic terms must also separately vanish and this can happen either through the parameters $\alpha$ and $\gamma$ taking special values or the fermionic coefficients having special relationships. (Remember that Grassmann var- 
iables are nilpotent.) However, the important conclusion of this analysis is that the parameter $\alpha$ can only have two distinct values, $\alpha=\frac{1}{2}$ or 1 . We also note that the resonance values in Eq. (4.18) correspond exactly to those of the nonlinear Schrödinger equation (bosonic) and correspond to $j$-values where bosonic variables can become arbitrary. $(j=-1$ corresponds to the arbitrariness in the location of the singularity surface.) The other four resonances, (either Eq. (4.19) or (4.20)), therefore, would correspond to the $j$ - values where the fermionic coefficients can become arbitrary. A detailed analysis of the recursion relations for $\alpha=\frac{1}{2}$ yields inconsistencies. We are, therefore, left only with $\alpha=1$. Furthermore, we are able to obtain consistent solutions in this case only for $\gamma=-1$. As is clear from Eqs. (4.18) and (4.20), there will be three arbitrary coefficients in this case at $j=0$. These correspond to

$$
\phi_{0} \quad, \quad \psi_{0}(t)=\beta(t) \phi_{0} \quad, \quad q_{0} p_{0}=\frac{1}{k}
$$

Here $\beta(t)$ is arbitrary. Similarly, there are three arbitrary coefficients at $j=3$ which can be identified with

$$
\phi_{3}(t) \quad, \quad \psi_{3}(t) \quad \text { and } \quad\left(q_{0} p_{3}-q_{3} p_{0}\right)
$$

The arbitrary bosonic coefficient at $j=4$ can be identified with

$$
q_{0} p_{4}-q_{4} p_{0}
$$

To conclude this section, let us note that if we choose $\phi_{3} \propto \phi_{0}$ and $\psi_{3} \propto \psi_{0}$, then some of 
the low order coefficients are determined from this analysis to be

$$
\begin{aligned}
q_{0} p_{0} & =\frac{1}{k} \quad \psi_{0}=\beta(t) \phi_{0} \\
\phi_{1} & =-\frac{i}{2} \xi_{t} \phi_{0} \quad \psi_{1}=\frac{i}{2} \xi_{t} \psi_{0} \\
q_{1} & =-\frac{i}{2} \xi_{t} q_{0}-i k q_{0} \phi_{0, t} \psi_{0} \\
p_{1} & =\frac{i}{2} \xi_{t} p_{0}+i k p_{0} \phi_{0, t} \psi_{0} \\
\phi_{2} & =\frac{i}{2} \phi_{0, t}-\frac{1}{12} \xi_{t}^{2} \phi_{0}-\frac{i k}{3} q_{0, t} p_{0} \phi_{0} \\
\psi_{2} & =-\frac{i}{2} \psi_{0, t}-\frac{1}{12} \xi_{t}^{2} \psi_{0}-\frac{i k}{3} q_{0, t} p_{0} \psi_{0} \\
q_{2} & =\frac{i}{6} q_{0, t}-\frac{1}{12} \xi_{t}^{2} q_{0}-\frac{k}{6} \xi_{t} q_{0} \phi_{0, t} \psi_{0} \\
p_{2} & =-\frac{i}{6} p_{0, t}-\frac{1}{12} \xi_{t}^{2} p_{0}-\frac{k}{6} \xi_{t} p_{0} \phi_{0, t} \psi_{0} \\
q_{3} p_{0}+q_{0} p_{3} & =\frac{1}{4 k} \xi_{t t}-\frac{1}{2}\left(\phi_{0, t t} \psi_{0}+\phi_{0, t} \psi_{0, t}\right)
\end{aligned}
$$

and so on. We emphasize here that the Painlevé analysis seems to select out $\alpha=1=-\gamma$ which is also the value we obtained in trying to construct the matrix Lax pair in sec. III.

\section{Zero Curvature Formulation:}

Much like the KdV equation, the nonlinear Schrödinger equation can also be written as a zero curvature with potentials belonging to $\mathrm{SL}(2, \mathbf{R})$ (or $\mathrm{SU}(2)$ ) [1,2]. In fact, the potentials

$$
\begin{gathered}
A_{1}=\left(\begin{array}{cc}
i \zeta & -i k^{1 / 2} q^{*} \\
i k^{1 / 2} q & -i \zeta
\end{array}\right) \\
A_{0}=\left(\begin{array}{cc}
-2 i \zeta^{2}-i k q^{*} q & -k^{1 / 2} q_{x}^{*} \\
-k^{1 / 2} q_{x} & 2 i \zeta^{2}+i k q^{*} q
\end{array}\right)
\end{gathered}
$$

give rise the field strength (curvature)

$$
F_{01}=\partial_{0} A_{1}-\partial_{1} A_{0}+\left[A_{0}, A_{1}\right]
$$


where $\partial_{0}=\frac{\partial}{\partial t}, \partial_{1}=\frac{\partial}{\partial x}$. Requiring the field strength to vanish yields the nonlinear Schrödinger equations of (2.1). In trying to formulate the supersymmetric nonlinear Schrödinger equation as a zero curvature, we recall that the supersymmetric KdV equation can be formulated as a zero curvature condition associated with the graded group OSp(2|1) $[12,13] \operatorname{OSp}(2 \mid 1)$ has five generators and in components the zero curvature condition has the form

$$
\partial_{0} A_{1}^{I}-\partial_{1} A_{0}^{I}+f^{I J K} A_{0}^{J} A_{1}^{K}=0
$$

where $I, J, K=1,2,3$ can be thought of as bosonic indices (the corresponding generators satisfy commutation relations) while $I, J, K=4,5$ can be thought of as fermionic indices (the corresponding generators satisfy anticommutation relations). From Eq. (5.1), we see that

$$
\begin{aligned}
& {\left[A_{1}^{1}\right]=\left[A_{1}^{2}\right]=\left[A_{1}^{3}\right]=1} \\
& {\left[A_{0}^{1}\right]=\left[A_{0}^{2}\right]=\left[A_{0}^{3}\right]=2}
\end{aligned}
$$

A simple dimensional analysis of Eq. (5.3) shows that

$$
\begin{aligned}
& {\left[A_{1}^{4}\right]=\left[A_{1}^{5}\right]=1} \\
& {\left[A_{0}^{4}\right]=\left[A_{0}^{5}\right]=2}
\end{aligned}
$$

Since the basic fermionic variables, $\phi$ and $\phi^{*}$, have canonical dimension $\frac{1}{2}$, the fermionic potentials must come multiplied by a dimensional parameter of dimension $\frac{1}{2}$. The supersymmetric equations, Eq. (2.3), on the other hand, do not involve any dimensional parameter. Therefore, the equations, if they can be derived, must hold for any value of the dimensional parameter. However, we note that in the limit of the vanishing dimensional parameter, the fermionic variables would drop out leading to an inconsistency. Thus, this simple argument shows that the supersymmetric nonlinear Schrödinger equation cannot be formulated as a zero curvature associated with $\operatorname{Osp}(2 \mid 1)$. It is an open question as to whether it can be expressed as a zero curvature associated with a different graded group. If it can be formulated as a zero curvature condition, through the standard discussions, it can also be obtained from a self-duality condition [16]. 
Even though we have not succeeded in formulating the equations as a zero curvature condition, we would like to point out the following. Consider the following potentials belonging to $\operatorname{OSp}(2 \mid 1)$.

$$
\begin{gathered}
A_{1}=\left(\begin{array}{ccc}
k \phi^{*} \phi & -i k^{1 / 2} q^{*} & -i \lambda k^{1 / 2} \phi^{*} \\
i k^{1 / 2} q & -k \phi^{*} \phi & i \lambda k^{1 / 2} \phi \\
i \lambda k^{1 / 2} \phi & i \lambda k^{1 / 2} \phi^{*} & 0
\end{array}\right) \\
A_{0}=\left(\begin{array}{ccc}
-i k\left(q^{*} q+\phi_{x} \phi^{*}-\phi \phi_{x}^{*}\right) & -k^{1 / 2} q_{x}^{*} & -\lambda k^{1 / 2}\left(\phi_{x}^{*}+i k^{1 / 2} q^{*} \phi\right) \\
-i k^{1 / 2} q_{x} & i k\left(q^{*} q+\phi_{x} \phi^{*}-\phi \phi_{x}^{*}\right) & -\lambda k^{1 / 2}\left(\phi_{x}-i k^{1 / 2} \phi^{*} q\right) \\
-\lambda k^{1 / 2}\left(\phi_{x}-i k^{1 / 2} \phi^{*} q\right) & \lambda k^{1 / 2}\left(\phi_{x}^{*}+i k^{1 / 2} q^{*} \phi\right) & 0
\end{array}\right)
\end{gathered}
$$

Here $[\lambda]=\frac{1}{2}$. The field strength

$$
F_{01}=\partial_{0} A_{1}-\partial_{1} A_{0}+\left[A_{0}, A_{1}\right]
$$

can be constructed in a straightforward manner and requiring this to vanish yields the equations

$$
\begin{aligned}
i q_{t}=- & q_{x x}+2 k\left(q^{*} q\right) q-2 k q \phi \phi_{x}^{*}+2 k q \phi_{x} \phi^{*}-2 k q_{x} \phi \phi^{*} \\
& +2 i \lambda k^{1 / 2} \phi_{x} \phi+2 \lambda k q \phi^{*} \phi \\
i \phi_{t}=- & \phi_{x x}+2 k\left(q^{*} q\right) \phi-2 k \phi \phi^{*} \phi_{x}
\end{aligned}
$$

We recognize Eq. (5.8) as identical to the fermionic equation of the supersymmetric equation for $\alpha=1=-\gamma$ given in Eq. (3.6). Even the bosonic equation (5.7) is a generalization of the bosonic equation in Eq. (3.6). The new set of equations in Eqs. (5.7) and (5.8) are no longer supersymmetric mainly because of the $\lambda$-dependent terms. However, one can think of them as a new fermionic extension of the nonlinear Schrödinger equation similar to the earlier construction $[17,18]$. A preliminary analysis shows that these set of equations have the Painlevé property and, therefore, are likely to be integrable. It is tempting 
to say that in the limit $\lambda \rightarrow 0$, the set of equations (5.7) and (5.8) reduce to Eq. (3.6) and, therefore, we have a zero curvature formulation for the special values $\alpha=1=-\gamma$. However, as we have argued when $\lambda \rightarrow 0$, fermionic variables drop out of the potential and, therefore, in some sense it is an improper limit and the zero curvature formulation of the supersymmetric nonlinear Schrödinger equation remains an open problem.

\section{The Hamiltonian structures:}

Bosonic integrable systems such as the KdV system are known to have biHamiltonian structures [19]. In fact, the biHamiltonian structures lead to recursion operators which in turn lead to a nice geometric interpretation of integrability in such systems [20,21]. The nonlinear Schrödinger equation is also known to have a biHamiltonian structure much like the KdV system $[1,19]$. In fact, if we define

$$
H_{1}=-\int d x\left(q_{x}^{*} q_{x}+k\left(q^{*} q\right)^{2}\right)
$$

and

$$
\left\{q\left(x_{1}, t\right), q^{*}\left(x_{2}, t\right)\right\}_{1}=i \delta\left(x_{1}-x_{2}\right)
$$

with all other Poisson brackets vanishing, then it is quite straightforward to check that the nonlinear Schrödinger equation (Eq. (2.1)) can be written as a Hamiltonian system,namely,

$$
\begin{gathered}
i q_{t}=i\left\{q, H_{1}\right\}_{1} \\
-i q_{t}^{*}=-i\left\{q^{*}, H_{1}\right\}_{1}
\end{gathered}
$$

Conventionally, the Hamiltonian structure in Eq. (6.2) is known as the first Hamiltonian structure.

Let us further note that we can also choose as a Hamiltonian for the system

$$
H_{2}=\int d x i\left(q^{*} q_{x}-q_{x}^{*} q\right)
$$


(Both $H_{1}$ and $H_{2}$ are conserved under the evolution of $q$ and $q^{*}$ given in Eq. (2.1).) If we now choose as the basic Poisson brackets of the theory

$$
\begin{aligned}
\left\{q\left(x_{1}, t\right), q\left(x_{2}, t\right)\right\}_{2} & =\frac{k}{2} q\left(x_{1}, t\right) q\left(x_{2}, t\right) \epsilon\left(x_{1}-x_{2}\right) \\
\left\{q\left(x_{1}, t\right), q^{*}\left(x_{2}, t\right)\right\}_{2} & =\frac{1}{2} \partial_{x_{1}} \delta\left(x_{1}-x_{2}\right)-\frac{k}{2} q\left(x_{1}, t\right) q^{*}\left(x_{2}, t\right) \epsilon\left(x_{1}-x_{2}\right) \\
\left\{q^{*}\left(x_{1}, t\right), q^{*}\left(x_{2}, t\right)\right\}_{2} & =\frac{k}{2} q^{*}\left(x_{1}, t\right) q^{*}\left(x_{2}, t\right) \epsilon\left(x_{1}-x_{2}\right)
\end{aligned}
$$

where $\epsilon\left(x_{1}-x_{2}\right)$ is the antisymmetric step function, then it is straightforward to show that the nonlinear Schrödinger equation can be written as a Hamiltonian system

$$
\begin{gathered}
i q_{t}=i\left\{q, H_{2}\right\}_{2} \\
-i q_{t}^{*}=-i\left\{q^{*}, H_{2}\right\}_{2}
\end{gathered}
$$

In other words, nonlinear Schrödinger equation is a biHamiltonian system and Eq. (6.5) describes the second Hamiltonian structure of the theory.

It is known (mainly from the study of the supersymmetric KdV system) that the two Hamiltonian structures of a bosonic integrable system do not generalize to the supersymmetric case $[6,22,23]$. In fact, for $\mathrm{KdV}$, the first Hamiltonian structure generalizes to superspace in the case of a supersymmetric theory that is not integrable while it is the second Hamiltonian structure that generalizes to the correct integrable supersymmetric theory [6]. While this is not a test of integrability, it would be interesting to study the analogous situation for the supersymmetric nonlinear Schrödinger equation.

Note that $H_{1}$ can be generalized readily to superspace as

$$
\begin{aligned}
H_{1}=\int d \mu[ & \frac{1}{2}\left(\left(D^{3} \Phi^{*}\right)\left(D^{2} \Phi\right)+\left(D^{3} \Phi\right)\left(D^{2} \Phi^{*}\right)\right)+\frac{k}{2}\left(\left(D \Phi^{*}\right)^{2} \Phi(D \Phi)\right. \\
& \left.\left.+(D \Phi)^{2} \Phi^{*}\left(D \Phi^{*}\right)\right)+c \Phi^{*} \Phi\left(\left(D^{2} \Phi\right)\left(D \Phi^{*}\right)-\left(D^{2} \Phi^{*}\right)(D \Phi)\right)\right]
\end{aligned}
$$

Here "c" is an arbitrary parameter and $d \mu=d x d \theta, \int d \theta=0$ and $\int \theta d \theta=1$. (This Hamiltonian reduces to Eq. (6.1) when the fermions are set equal to zero.) Similarly, the first Hamiltonian structure in Eq. (6.2) can be generalized to superspace as

$$
\left\{\Phi\left(x_{1}, \theta_{1}, t\right), \Phi^{*}\left(x_{2}, \theta_{2}, t\right)\right\}_{1}=-i D_{1}^{-1} \Delta_{12}=-i D_{2}^{-1} \Delta_{12}
$$


with all other Poisson brackets vanishing. Here

$$
D_{1}=\frac{\partial}{\partial \theta_{1}}+\theta_{1} \frac{\partial}{\partial x_{1}}
$$

and

$$
D_{1}^{-1}=\partial_{x_{1}}^{-1} D_{1}
$$

with

$$
\Delta_{12}=\delta\left(x_{1}-x_{2}\right) \delta\left(\theta_{1}-\theta_{2}\right)=\delta\left(x_{1}-x_{2}\right)\left(\theta_{1}-\theta_{2}\right)
$$

Requiring the superspace equations (see Eq. (2.10)) to be Hamiltonian of the form

$$
\begin{gathered}
i \Phi_{t}=i\left\{\Phi, H_{1}\right\}_{1} \\
-i \Phi_{t}^{*}=-i\left\{\Phi^{*}, H_{1}\right\}_{1}
\end{gathered}
$$

yields

$$
c=-1 \quad \gamma=0 \quad \alpha=1
$$

We see that the generalization of the first Hamiltonian structure selects out a set of values of the parameters which does not satisfy the Painlevé property much like the supersymmetric $\mathrm{KdV}$ case [6].

The generalization of the Hamiltonian in Eq. (6.4) to superspace is simpler and has the form

$$
H_{2}=\int d \mu i\left(\left(D^{3} \Phi^{*}\right) \Phi-\left(D^{3} \Phi\right) \Phi^{*}\right)
$$

The generalization of the second Hamiltonian structure in Eq. (6.5) needs a little bit of work but can be determined to be

$$
\begin{aligned}
\left\{\Phi\left(x_{1}, \theta_{1}, t\right), \Phi\left(x_{2}, \theta_{2}, t\right)\right\}_{2} & =k \Phi\left(x_{1}, \theta_{1}, t\right) \Phi\left(x_{2}, \theta_{2}, t\right) D_{1}^{-1} \Delta_{12} \\
\left\{\Phi\left(x_{1}, \theta_{1}, t\right), \Phi^{*}\left(x_{2}, \theta_{2}, t\right)\right\}_{2} & =-\frac{1}{2} D_{1} \Delta_{12}-k \Phi\left(x_{1}, \theta_{1}, t\right) \Phi^{*}\left(x_{2}, \theta_{2}, t\right) D_{1}^{-1} \Delta_{12} \\
\left\{\Phi^{*}\left(x_{1}, \theta_{1}, t\right), \Phi^{*}\left(x_{2}, \theta_{2}, t\right)\right\}_{2} & =k \Phi^{*}\left(x_{1}, \theta_{1}, t\right) \Phi^{*}\left(x_{2}, \theta_{2}, t\right) D_{1}^{-1} \Delta_{12}
\end{aligned}
$$


Requiring that the superspace equations be Hamiltonian with respect to Eqs. (6.14) and (6.15), namely,

$$
\begin{gathered}
i \Phi_{t}=i\left\{\Phi, H_{2}\right\}_{2} \\
-i \Phi_{t}^{*}=-i\left\{\Phi^{*}, H_{2}\right\}_{2}
\end{gathered}
$$

yields

$$
\begin{aligned}
i \Phi_{t} & =-D^{4} \Phi+2 k\left(D \Phi^{*}\right)(D \Phi) \Phi+2 k \Phi^{*} \Phi\left(D^{2} \Phi\right) \\
-i \Phi_{t}^{*} & =-D^{4} \Phi^{*}+2 k(D \Phi)\left(D \Phi^{*}\right) \Phi^{*}+2 k \Phi \Phi^{*}\left(D^{2} \Phi^{*}\right)
\end{aligned}
$$

Comparing with Eq. (2.10), we note that this selects out the values of the parameters to be

$$
\alpha=1=-\gamma
$$

These are, of course, the values for which the Painlevé analysis goes through. Thus, once again, we see, as in the case of the supersymmetric KdV equation, that the generalization of the second Hamiltonian structure selects out the values of the parameters that are consistent with the Painlevé analysis.

\section{Conclusion:}

We have studied the supersymmetric nonlinear Schrödinger equation systematically. We have shown that various conventional tests of integrability select out a theory where there is no arbitrariness in the parameters. (This is consistent with one set of values Eq. (2.11) in ref. 4 assuming a misprint.) We have not succeeded in obtaining a zero curvature formulation of this system based on the group $\operatorname{OSp}(2 \mid 1)$. However, we obtain a fermionic generalization of the theory which also appears to possess the Painlevé property. We have examined the generalization of the Hamiltonian structures to superspace. We find, much like the supersymmetric KdV equation, that the generalization of the first Hamiltonian structure selects out a set of values for the parameters which do not satisfy the Painlevé property whereas the generalization of the second Hamiltonian structure yields values of the parameter consistent with the Painlevé analysis. In light of our analysis, the second 
set of values of the parameters containing a free parameter (see Eq. (2.12)) obtained in ref. 4 from the study of the prolongation structure remains a puzzle.

This work was supported by U.S. Department of Energy Grant No. DE-FG-0291ER40685. One of us (J.C.B.) would like to thank CNPq, Brazil for financial support. 


\section{References:}

1. L. D. Faddeev and L. A. Takhtajan, Hamiltonian methods in the theory of solitons (Springer, Berlin, 1987).

2. A. Das, Integrable models (World Scientific, Singapore, 1989).

3. M. J. Ablowitz and P. A. Clarkson, Solitons, nonlinear evolution equations and inverse scattering (Cambridge, New York, 1991).

4. G. H. M. Roelofs and P. H. M. Kersten, J. Math. Phys. 33, 2185 (1992).

5. F. B. Estabrook and W. D. Wahlquist, J. Math. Phys. 17, 1293 (1976).

6. P. Mathieu, J. Math. Phys. 29, 2499 (1988).

7. A. Das, W-J. Huang and S. Roy, Phys. Lett. 157A, 113 (1991).

8. M. J. Ablowitz, A. Ramani and H. Segur, J. Math. Phys. 21, 715 (1980).

9. J. Weiss, M. Tabor and G. Carnevale, J. Math. Phys. 24, 522 (1984).

10. S. S. Chern and C. K. Peng, Manuscr. Math. 28, 207 (1979).

11. M. Crampin, F. A. Pirani and D. C. Robinson, Lett. Math. Phys. 2, 15 (1977).

12. A. Das and S. Roy, J. Math. Phys. 31, 2145 (1990).

13. A. Das, W-J. Huang and S. Roy, Int. J. Mod. Phys. 15A, 3447 (1992).

14. V. E. Zakharov and A. B. Shabat, Sov. Phys. JETP 34, 62 (1972).

15. See for example, "Group Theory in Physics", vol. 3 by J. F. Cornwell, Academic Press (1989).

16. A. Das and C. A. P. Galvão, Mod. Phys. Lett. A8, 1399 (1993); J. C. Brunelli and A. Das, Davey-Stewartson equation from a zero curvature and a self-duality condition, University of Rochester preprint UR-1332 (1993) (also hep-th/9312070) and references therein.

17. P. P. Kulish, ICTP, Trieste preprint IC/85/39, 1985.

18. R. A. Chowdhury and M. Naskar, J. Math. Phys. 28, 1809 (1987).

19. F. Magri, J. Math. Phys. 19, 1156 (1978).

20. S. Okubo and A. Das, Phys. Lett. 209B, 311 (1988). 
21. A. Das and S. Okubo, Ann. Phys. (N.Y.) 190, 215 (1989).

22. W. Oevel and Z. Popowicz, Comm. Math. Phys. 139, 441 (1991).

23. J. M. Figueroa-O'Farrill, J. Mas and E. Ramos, Leuven preprint KUL-TF-91/19 (1991). 